as those of mags. $9^{\cdot} 1-9 \cdot 5$. This correlation appears to indicate that the faintest stars and those of the 9th or loth mag. probably form part of one system, and are at nearly the same distance from us. At the same time, some stars brighter than 9th wag. seem to be intimately associated with the Milky Way.

The hypothesis of an annular system, relatively isolated from the central part of the great galactic system, is regarded as not incompatible with the distrihution of stars which he bas found. For if, at nearly the same distance from us, stars vary so much in size or intinsic brightness as to give magnitudes ranging from 9 to 15 , there seems no reason why some should not be of greater brightness than gth mag.

There is nothing to prove, however that the various parts of the Milky Way are at an equal distance from us, nor even that it may be an enclosed ring. It does not appear improbable that subsequent researches may show the existence of one or several spirals emanating from a central accumulation, and recurving so as to form a nearly annular system, or one consisting of nearly concentric rings. However it may be, Mr. Easton's results seem to is.dicate that the portion of the Milky Way accessible to our weans of observaition, has but little thickness in relation to the diameter.

In a paper on the same subject (Knozuledge, February), Mr. Maunder finds it difficult to re,ist the conclusion that the "dark lan: s" of the Milky Way are really region, of barrennese, and regards these features as indications of a process of condensation going on in the stellar as well as in the sebulous matter.

The System of Algol.-Quite recently (NATURE, vol. xlv. p. 446) Mr. Chandler credited Algol with an obscure companion in addition to that which was recognised by Goodricke, and the existence of which has been fully confirmed by the in vestigations of Pickering and Vogel. Mr. Chandler's conclusions were based on a discussion of the systematic ir regularities of the epochs of minima, and were apparently confirmed by a later discussion of the proper motion of Algol itself (NATURE, vol. xlix. p. 349). The evidence of irregular proper motion, however, is not regarded as conclusive by some authorities, and M. Tisserand, the Director of the Paris Observatory, is ap parently one of the unconvinced. He has therelore attempted to find some other explanation of the phase variations (Comptes. Rendus, January 21, 1895), and the result is to show that they can be simply and sufficiently explained by supposing a single dark companion moving in an elliptic, instead of a circular, orbit; and, in addition, that the bright star exhibits a sensible polar compression. The result of this departure from the spherical form would be a movement of the periastron point, and this would explain the apparent irregularities.

Assuming that the plane of the orbit is coincident with the equator of Algol, its eccentricity is found to be 0.132 , and the polar diameter is shorter than the equatorial by $\frac{1}{2} \frac{1}{6} 6$.

The consequences of these conditions would be a very slight variation of minimum brightness in the long period of 140 years, and an entirely negligible difference in the time of passage to minimum and recovery of normal brightness. The total duration of the eclipse, however, will vary very considerably. Taking the mean epochs 1800 and $188_{4}$ for the observations of Wurm and Schonfeld respectively, M. Tisserand finds that the duration would be increased in this time by 1 ' 63 hours. Since the times given by these observers are $6 \cdot 5 \mathrm{~h}$. and $9 \cdot 0 \mathrm{~h}$. respectively, M. Tisserand is entitled to regard this as confirmation of his hypothesis. He points out the importance of spectroscopic observations at short intervals from miminum, in connection with his explanation.

The irregularities in $U$ Ophiuchi and $U$ Cephei are pro bably to be explained in the same way.

\section{THE EXPLOSIVE NATURE OF THE SODIUM AND POTASSIUM DERIVATIVES OF NITROMETHANE.}

SOME additional information of an interesting character con cerning the extremely explosive sodium and potasium compounds of nitromethane, is contributed to the current Berichte of the German Chemical Society by Prof. Zelinsky of Mo-cow. A sholt time ago Prof. Victor Meyer described (Berichte, 27, I601) a mode of preparing the sodium cumpound $\mathrm{CH}_{2} \mathrm{NaNO}_{2}$ in a state of purity. The process consists in diluting a quan'ity of nitromethane, $\mathrm{CH}_{3} \mathrm{NO}_{2}$, with ether and treating the liquid with a solution of sodium in alcohol, when the sodium compound is precipitated. The precipitate requires to be washed with ether, and is then dried over oil of vitriol; the NO. I 318 , VOL. 51$]$ dry compound thus obtained affords numbers on analysis agreeing with the anhydrous formula above given. In a former method of preparation described by Prof. Meyer, alcoholic soda was employed as precipitant, but the sodium nitromethane obtained invariably contained either water or alcohol; the usc of sodium ethylate affords it anhydrous. Even the hydrated compound first isolated proved to be explosive; but upon placing a small quantity of it upon a wa'ch-glass, and warming over a water-bath, in a short time it became suddenly converted into the anbydrous cump und which immediately exploded with great violence. When a small quantity of the anhydrous compound prepared by use of sodium ethylate was placed in a test-tube, gently cowpresied, and then warmed, an explosion of so violent a nature occurred that the test-tube was completely pulverised.

Prof. Z linsky has recently had occasion to prepare consider able quantities of sodium and potas-ium nitromethane, and has had the opportunity of testing and observing their explosive power upon a larger scale. He appears to have adopted essentially the same process for the preparation of sodium nitro. methane as that described by Prof. Meyer, employing an alcoholic solution of sodium ethylate as precipitating reagent. Being desirous of obtaining the sudium compound periectly anhydrous, an attempt was made to achieve this object by use of the water bath, but for the sake of precaution only about a gram of the substance was employed as a preliminary test of the efficacy of this method of dehydration. It was fortunate that such was the case, for within five minutes an explosion of so violent a nature occurred, that the watch-glas; upon which the compound was supported was reduced to powder, and the water-bath considerably injured. In order to demonstrate the explosive nature of this compound without danger upon the lecture table, Pro'. Zelinsky recommends the following experiment :-A thick clock-glass, or better a stout metal plate, is sprinkled with small drops of water, and a very small piece of sodium nitromethane dropped upon it. After a $\mathrm{f} \in \mathrm{w}$ seconds, provided the amount of water has not been excessive, a deafening detonation occurs, with production of flame and projection of a thick cloud of smoke. The experiment may be varied by placing the substance upon the perfectly dry plate, and invoking its explosion by means of a smart blow with a hard object.

M Nef has previously (Ann. der Chemie, 280, 273) described several of the metallic derivates of the nitroparaffins, and has referred to the instability of the sodium compound, and the possibility of occasional explosions. Prof. Zelinsky now supplements this statement by remarking that an explosion always results from the contact of the dry sodium compound with a minute quantity of water. One of his assistants upon one occasion incautiously placed about five grams of sodium nitromethane in a glass vessel whose surface happened to be moist, with the result that a terrific explosion instantly occurred, which shattered every piece of apparatus upon the table, and the atmospheric wave produced occasioned the sudden extinction of the whole of the gas flames in the laboratory. The assistant fortunately escaped more than trifing injury, but a second such occurrence might have a very different result. This incident will doubtless serve to emphasise the great precaution which is necessary in handling these compounds.

The potassium compound, $\mathrm{CH}_{2} \mathrm{KNO}_{2}$, has been prepared in a similar manner, and found to be even more unstable than the sodium compound, exploding at the ordinary temperature shortly after its isolation. It separates upon the addition of the potassium ethylate in well defined crystals. The crystalline form, however, soon disappears, and upon rapidly transferring 10 a filter, an explosion invariably occurs as soon as the compound becomes drained free of most of the mother liquor. The intability of the potassium compound at the ordinary temperature may also be readily demonstrated upon the lecture table. It is, of course, necessary to prepare it freshly on the spot, because of the impossibility of preserving it for any length of time. An ethereal solution of nitromethane is mixed with a solution of potassium ethylate in alcohol, the supernatant liquid rapidly decanted from the precipitate produced, the latter dried as quickly as possible between filter-paper, and left quietly resting upon the paper. After a few minutes the substance explodes with a loud detonation.

These experiments will serve to indicate the extreme instability of the alkali-metal derivatives of nitromethane, and the violence of the explosions produced by their disruption.

A. E. TutTon. 